\title{
An Assessment of Alternative Therapeutic Options for the Treatment of Prolonged Zoonotic Fungal Infections in Companion Animals
}

\author{
Meade E1, Rawe $S^{2}$, Fowley $\mathrm{C}^{1}$, Slattery $\mathrm{MA}^{3}$ and Garvey $\mathbf{M}^{1,2,3 *}$ \\ 1Department of Life Science, Institute of Technology, Ireland \\ ${ }^{2}$ School of Chemical and Pharmaceutical Science, Dublin Institute of Technology, \\ Ireland \\ ${ }^{3}$ Mark Anthony Slattery, Veterinary Practice, Ireland
}

Research Article

Volume 4 Issue 3

Received Date: July 01, 2019

Published Date: July 25, 2019

DOI: $10.23880 /$ oajmb-16000149

*Corresponding author: Mary Garvey, Department of Life Science, Institute of Technology Sligo, Ash lane, Sligo, Ireland, Tel: 071 9305529; Email: Garvey.Mary@itsligo.ie

\section{Abstract}

Zoonotic disease is an ongoing issue, becoming more prominent as more microbial species display antimicrobial resistance. The role of companion animals in society is also increasing as therapy dogs become the mainstay for many special needs persons. In order to ensure public health safety, it is essential to determine the extent of resistance amongst zoonotic pathogens and where possible to suggest novel treatment options to counteract such resistance. Fungal species are increasingly recognised as the causative agent in numerous incidents of canine morbidity. Therefore, the development of new, safe and effective chemotherapeutic agents is essential to prevent and control mycotic disease. Chronic incidents of cutaneous candidiasis as diagnosed in the cases used for this study are particularly high risk for zoonotic transmission. Studies described herein evaluate the resistance of these canine isolates of Candida species to common antifungal agents and identify levels of resistance using recognised in vitro methods. Results show high levels of resistance to amphotericin B, fluconazole and caspofungin for Candida albicans and Candida krusei isolates. This clinical resistance was more prominent in isolated species compared to control species. Novel compounds namely phendione and Roussin Black salts show promising antifungal activity with minimum inhibitory concentrations determined.

Keywords: Zoonotic; Fungal; Fatal; Inactivation; Resistance

\section{Introduction}

Anthropozoonosis (zoonotic) diseases are transmitted from animal to human hosts often resulting in pathogenesis. Zooanthroponosis however, describes diseases which transmit from human to animal hosts where, both are of increasing importance as multidrug resistant infections are becoming the mainstay. Indeed, zooanthroponosis may contribute to the increasing rates of mycosis in companion animals as pathogens transmit from close contact. Such diseases include infections 


\section{Open Access Journal of Microbiology \& Biotechnology}

resulting from numerous microbial species of bacterial (Staphylococcus, Streptococcus), viral (influenza A virus), fungal (Candida) and protozoal (Cryptosporidium) origin. Previously, the largest incidences of morbidity and mortality in companion animals were from bacterial and viral infections, however fungal infections have become increasingly prevalent [1]. So much so, dermal and systemic fungal infections in companion animals currently represent a routine problem for veterinarians. Furthermore, fungal infections (mycosis) in humans are common and often fatal, by end of the 1990s fungal infections had become the seventh leading cause of human morbidity resultant from infectious disease [2]. Such mycotic disease may be acute, sub-acute or chronic in nature manifesting as dermatitis, pneumonia, meningitis or osteomyelitis. Fungal pathogens are a major concern for immunocompromised persons such as AIDS patients or persons on immunosuppressive therapy. Epidemiology studies show that patients with advanced AIDS harbour fluconazole-resistant $C$. albicans in their oral cavities [3]. For animal species however, the recognition and identification of fungal disease is often only a post mortem discovery [4]. The treatment and control of invasive fungal infections in animals traditionally relied on the use of amphotericin B with the azoles, fluconazole and itraconazole being introduced in the last decade. Drug resistance in fungal species such as Candida is an emerging problem with resistance to these antifungals a common occurrence in infected animals [5]. Furthermore, amphotericin B has issues with animal toxicity, poor absorption from the gastrointestinal tract (GIT), drug/drug, and drug/food interactions, while the azole drugs also present with erratic absorption patterns [6] making these drugs less than efficient oral antifungal agents. Consequently, the prognosis for infected animals remains poor with mortality rates exceeding $80 \%$. This drug and multi drug resistance is also seen in fungal biofilm communities due to their slow growth rate and the reduced rate of therapeutic penetration into the biofilm matrix [7]. The formation of biofilm structures in the chronic stages of infection in dogs has been identified in many chronic fungal infections such as periodontitis, otitis media and endocarditis [8]. Therefore, there is a need for novel antifungal agents for the treatment of systemic and dermal fungal infections in companion animals and the prevention of zoonotic transmission. Such drugs should have antifungal activity, improved GIT absorption while having improved biocompatibility to the animal itself in comparison to the antifungal therapeutics currently in use. Given the pathogenic and zoonotic potential of these microorganisms, this study aims to assess the antifungal activity of a range of synthesised chemotherapeutic agents on veterinary isolates of Candida species. The findings of this study may help identify novel antifungal therapeutics for use in animal cases of candidiasis. Candida species under study were isolated from infected dogs presenting with prolonged dermal or systemic infections.

\section{Methodology}

Amphotericin B (Amp B), fluconazole and caspofungin were sourced from Sigma Aldrich, Dublin, Ireland. All therapeutic agents were made to stock solutions by dissolving the drug powder in adequate volumes of dimethyl sulfoxide (DMSO) (wgt/vol) with the exception of Roussin Black Salt (RBS) which is a polar compound and therefore, dissolves in sterile phosphate buffered saline (Sigma Aldrich, Dublin, Ireland). Once completely dissolved with no precipitation evident, working concentrations of all test agents where then made by diluting the stocks in sterile $0.01 \mathrm{M}$ phosphate buffer, containing $0.0027 \mathrm{M} \mathrm{KCL}$ and $0.137 \mathrm{MNaCl}$ at a pH of 7.4 (PBS).

\section{Drug synthesis and Characterization}

Reagents and solvents for synthesis of MIT, phendione and Roussin's black salt (RBS) were purchased from Sigma Aldrich, Fisher and Apollo Scientific and were used as supplied. NMR spectra were measured using a Bruker Avance III (400 MHz). Chemical shifts $(\delta)$ are expressed in parts per million (ppm) relative to TMS (tetramethylsilane, internal standard). Coupling constants (J) are expressed in Hertz, multiplicities of signals are reported as doublets (d) and doublet of doublets (dd). High resolution mass spectrometry was performed in University College Dublin by Kevin Conboy. Melting points were measured on a Stuart MP/SMP10/ melting point apparatus and are uncorrected. Infrared (IR) spectra were recorded on a Perkin Elmer Spotlight 400N FT-IR spectrometer using a UATR accessory.

Mitonafide (MIT, 2-(2-(dimethylamino)ethyl)-3-nitro1H-benzo[de]isoquinoline-1,3(2H)-dione): To a mixture of 3-nitro-1,8-naphthalic anhydride (300 mg, $1.23 \mathrm{mmol}$ ) in toluene $(24 \mathrm{~mL})$ in a round bottomed flask equipped with a Liebig condenser was added ethanol (6 $\mathrm{mL})$, followed by $\mathrm{N}, \mathrm{N}$-dimethylethylenediamine $(161 \mu \mathrm{L}$, $1.46 \mathrm{mmol}$ ). The mixture was heated under reflux for 40 minutes, allowed to cool to room temperature, filtered and the volatiles removed in vacuo. The resulting dark brown residue recrystallised from hot ethanol $(10 \mathrm{~mL})$, to 


\section{Open Access Journal of Microbiology \& Biotechnology}

afford the pure product as a red-brown solid (140 mg, 36 $\%)$.

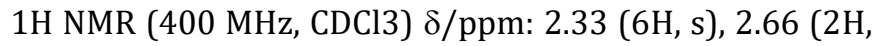
$\mathrm{t}, \mathrm{J}=6.8 \mathrm{~Hz}), 4.34(2 \mathrm{H}, \mathrm{t}, \mathrm{J}=6.8 \mathrm{~Hz}), 7.93(1 \mathrm{H}, \mathrm{dd}, \mathrm{J}=8.4$ and $7.4 \mathrm{~Hz}), 8.42(1 \mathrm{H}, \mathrm{dd}, \mathrm{J}=8.4$ and $0.8 \mathrm{~Hz}), 8.77(1 \mathrm{H}, \mathrm{dd}$, $\mathrm{J}=7.4$ and $0.8 \mathrm{~Hz}), 9.12(1 \mathrm{H}, \mathrm{d}, \mathrm{J}=2.2 \mathrm{~Hz}), 9.29(1 \mathrm{H}, \mathrm{d}, \mathrm{J}=$ $2.2 \mathrm{~Hz})$; 13C NMR (100 MHz, CDCl3) $\delta / \mathrm{ppm:} \mathrm{38.6,} \mathrm{45.8,}$ 56.9 (C-2), 123.2, 124.3, 124.7, 128.9, 129.1, 130.2, 131.0, 134.5, 135.6, 146.3, 162.6, 163.2; umax/cm-1 (ATR): 3080, 2779, 1706, 1662, 1598, 1541, 1509, 1435, 1417, 1344, $1327,1290,1238,1173,1159,1142,1075,1049,1019$, 963, 942, 912; HRMS (m/z, ES): calcd for C16H16N3O4 $[\mathrm{M}+\mathrm{H}]+314.1141$, found 314.1131; Melting point: 139$140^{\circ} \mathrm{C}$ (Lit. $139-140^{\circ} \mathrm{C}$ ) [9].

Phendione (1,10-Phenanthroline-5,6-dione): 1,10Phenanthroline $(5.010 \mathrm{~g}, 27.8 \mathrm{mmol})$ was dissolved in concentrated sulfuric acid $(30 \mathrm{~mL})$. Sodium bromide $(2.520 \mathrm{~g}, 24.5 \mathrm{mmol})$ was added portion-wise followed by concentrated nitric acid $(15 \mathrm{~mL})$. The resulting dark brown solution was heated at reflux for 5.5 hours. The reaction mixture was allowed to cool to room temperature and was poured over ice $(500 \mathrm{~mL})$. It was neutralised with aqueous sodium hydroxide $(400 \mathrm{~mL}, 3$ $\mathrm{M})$, and extracted with DCM (3 x $100 \mathrm{~mL})$, dried over anhydrous sodium sulfate and the volatiles were removed to give a brown amorphous solid. Recrystallisation from toluene and methanol ( $4: 1)$ gave the product as an orange solid (1.95 g, 33 \%). 1H NMR (400 MHz, DMSOd6) ? /ppm: $8.98(\mathrm{dd}, 2 \mathrm{H}, \mathrm{J}=4.8$ and $2.0 \mathrm{~Hz}), 8.38(\mathrm{dd}, 2 \mathrm{H}, \mathrm{J}=7.6$ and $2.0 \mathrm{~Hz}), 7.66(\mathrm{dd}, 2 \mathrm{H}, \mathrm{J}=7.6 \mathrm{~Hz}$ and $4.8 \mathrm{~Hz}) ; 13 \mathrm{C} \mathrm{NMR}$

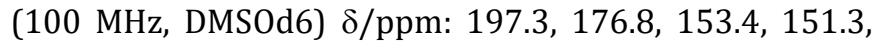
134.7, 128.1, 124.2; vmax/ $\mathrm{cm}^{-1}$ (ATR): 1686, 1566, 1437, $1379,735,720$; Melting point: $260-261^{\circ} \mathrm{C}$ (Lit. $260^{\circ} \mathrm{C}$ ).

Roussin black salt: Sodium Nitrite $7.2 \mathrm{~g}(0.10 \mathrm{~mol})$ wash charged to $33 \mathrm{~mL}$ of distilled water and stirred until dissolved. In a separate flask $8 \mathrm{~mL}$ of a $22 \%$ solution of Ammonium sulphide was added to $24 \mathrm{~mL}$ of distilled water. The resulting solutions were added together in a three neck flask resulting in a pale yellow solution. This was heated at reflux until the solution turned a dark cherry-red. A Ferrous Sulphate solution was prepared by adding Iron Sulphate heptahydrate $16 \mathrm{~g}(0.05 \mathrm{~mol})$ and $120 \mathrm{~mL}$ of distilled water. This solution was mixed until the solid had dissolved to give a pale green-blue solution. Once the original solution had turned a dark cherry red the iron sulphate solution was charged. The resulting solution turned black instantly. The solution was heated and stirred throughout. Ammonium Hydroxide 22\% 20 $\mathrm{mL}$ was then added in small portions while stirring. Once this addition was complete the solution was heated at reflux for $10 \mathrm{~min}$. The solution was then filtered hot with the filtrate retained. The resulting black solution was allowed to stand at room temperature overnight. The solution was then filtered and a black crystalline solid was collected and dried under vacuum to give $1.00 \mathrm{~g}(1 \mathrm{mmol})$ of $\mathrm{NH}_{4}\left[\mathrm{Fe}_{4} \mathrm{~S}_{3}(\mathrm{NO})\right.$,]. The resulting compound was used without further purification. vmax/ $\mathrm{cm}^{-1}\left(\mathrm{~cm}^{-1}\right)$ 1780, $1740,1700,1605 \mathrm{~cm}^{-1}$. Melting point: $198^{\circ} \mathrm{C}$ (Lit. 198 to $\left.200^{\circ} \mathrm{C}\right)$.

\section{Animal Morbidity and Clinical Symptoms}

Canine patient 1: an intact female 4-year-old fox terrier cross displaying chronic pruritus for $>1$ month, erythema along the ventral abdomen with musty odour evident. Also presented with a circular patch of inflamed skin with an oily discharge in a large area over the pelvis and an oily coat extending along the back to the neck. Very swollen, painful and discharging paws which were also very pruritic. Initial treatment consisted of malaseb wash (miconazole and chlorhexidine) (Dechra Veterinary Products, Uldum, Denmark) twice weekly and $250 \mathrm{mg}$ of cefalexin (antibiotic) twice daily for 3 weeks. Skin scrape using a blunted scalpel blade and microbial culture identified Candida krusei dermal infection via selective agars and PCR amplification. Diagnosis, chronic cutaneous candidiasis with the zoonotic causative agent Candida krusei.

Canine patient 2: 6-year-old intact Jack Russell terrier with similar but not identical presenting signs. Symptoms include severe chronic pruritis with "greasy" skin along the back and inflamed skin along ventrum and legs. Skin flaking excessively and similar "musty" odour evident. Initially prescribed amoxicillin $(12.5 \mathrm{mg} / \mathrm{kg})$ with clavulanate acid $(3.124 \mathrm{mg} / \mathrm{kg})$ and prednisolone twice daily and received fipronil, s-methopene (flea treatment) followed by imidacloprid and moxidectin (topical flea and mange treatment). No alleviation of symptoms occurred. Treated with topical neomycin (antibiotic), triamcinolone (glucocorticoid) and nystatin (antifungal) once daily for 2 weeks and washed with malaseb twice weekly for 6 weeks. Skin scrape and microbial culture identified Candida albicans via selective agars and PCR identification. Diagnosis of cutaneous candidiasis with the zoonotic Candida albicans as the causative agent.

Canine patient 3: 4-year-old terrier cross presenting with pyometra and nephritis, dull, pale, diarrhoeic and foul smelling urine. Initial treatment prescribed 10 days of marbofloxacin (2 $\mathrm{mg} / \mathrm{kg}$ daily) and amoxicillin with 


\section{Open Access Journal of Microbiology \& Biotechnology}

clavulanic acid, also received rehydrating drip (IV) for the first 3 days. Urine sample and microbial culture identified Candida krusei via selective agars (confirmed by PCR). Diagnosis kidney infection with the zoonotic Candida krusei.

\section{Fungal Isolation, Identification, Culture and Maintenance}

Collected samples of infection (skin scrapes) were inoculated in sabouraud dextrose broth (Cruinn Diagnostics, Dublin, Ireland) and incubated at $30^{\circ} \mathrm{C}$ for up to 72 hours before streaking onto sabouraud dextrose agar (Cruinn Diagnostics, Dublin, Ireland). In the case of the prolonged canine kidney infection, a urine sample was obtained and inoculated onto sabouraud dextrose agar followed by incubation at $30^{\circ} \mathrm{C}$ for up to 72 hours. Individual colonies were streaked for isolation and Candida species identified based on their morphological characteristics, biochemical profile and growth on CHROMagar-Candida (CHROMagar, Paris). As CHROMagar medium includes chromogenic substrates, at least 48hour incubation period is necessary for the full colour development of Candida spp., with C. albicans isolate displaying green pigmented colonies and C. krusei isolates appearing as fuzzy, pink coloured colonies. Identity was confirmed via colony polymerase chain reaction (PCR). Strains were stored and cultured in sabouraud dextrose broth/agar at $30^{\circ} \mathrm{C}$ and identity confirmed via gram stain prior to each experimental set up.

PCR identification: A single Candida colony (DNA template) was picked from a 48-hour culture using a sterile micropipette tip and suspended in $100 \mu \mathrm{l}$ sterile deionized water. Fungal primers ITS1-F 5'-CTT GGT CAT TTA GAG GAA GTA A-3' and ITS4 5'TCCTCCGCTTATTGATATGC-3' (Sigma Aldrich, Dublin, Ireland) were used for direct amplification of intergenic spacer regions (ITS) of Candida rDNA. Direct colony PCR was performed in a total reaction volume of $20 \mu \mathrm{l}$, containing $17 \mu \mathrm{l}$ red Taq 1.1x master mix (VWR, Dublin, Ireland) $1 \mu \mathrm{l}$ ITS1F, $1 \mu \mathrm{l}$ ITS4 and $1 \mu \mathrm{l}$ of selected colony suspension. DNA amplification was performed in a thermo cycler (VWR, Dublin, Ireland) using the recommended parameters. Clean-up and gene sequencing of PCR products was completed by Source Bioscience (Waterford, Ireland).

\section{Scanning Electron Microscopy of Candida Isolates}

SEM was used to examine cell morphological of Candida species and to confirm species identification and cell cycle stage. Untreated skin isolates of $C$. albicans and
C. krusei were prepared by aseptically transferring a single colony into $10 \mathrm{ml}$ sterile sabouraud broth, followed by incubation at $30^{\circ} \mathrm{C}$ under rotary conditions (125 rpm). After 24-hour incubation period, cells were harvested via centrifugation (4000rpm, 15min) and washed with sterile PBS prior to fixing with formalin. Yeast cells were subsequently dehydrated by a sequential immersion $(10$ min) in water/ethanol $(50,70,80,90,100 \%)$ and ethanol/HMDS (50, 75, 100\%), and left to dry overnight in a desiccator at room temperature. Samples were then sputter-coated with gold and SEM imaged (Hitachi S4800).

\section{Antifungal Testing}

Antifungal susceptibility patterns of isolated Candida strains to amphotericin, caspofungin, fluconazole, mitonafide, phendione and Roussin black salt were assessed via disc diffusion (Kirby-Bauer) method, with the most effective drug concentrations selected for further study in the inhibition of growth assay. Two reference strains, Candida krusei ATCC 14243 and Candida albicans ATCC 10231, were included in each test for comparative sensitivity analysis to disease isolates. In addition, the effect of DMSO on Candida growth was evaluated by testing the different concentrations without the antifungal agent to negate the effect of DMSO induced toxicity, as it was used for drug dissolution.

Kirby Bauer assay: The Kirby-Bauer assay was performed to evaluate the sensitivity or resistance of isolated Candida spp. to all test agents. A test inoculum of each strain was prepared from a pure culture grown in sabouraud broth for 16 hours to enter the log phase of growth. Test samples were centrifuged at 10,000 rpm for 10 minutes and the pellet re-suspended in sterile phosphate buffered saline and a serial dilution performed to determine a viable cell count. All fungal cell densities were adjusted to $1 \times 10^{6} \mathrm{cfu} / \mathrm{ml}$ in sterile PBS to maintain osmotic normality of the cells. Subsequently, $300 \mu \mathrm{L}$ of this microbial suspension were transferred onto triplicate sabouraud agar plates and spread with a sterile L-shaped spreader (Cruinn Diagnostics) to ensure even disruption across the agar surface. Agars were prepared per manufacturer's instructions in deionized water at room temperature and poured to a depth of $4 \mathrm{~mm}$. Immediately before inoculation, media was checked to ensure it was moist but free of water droplets on the agar surface and the petri dish lids. Antimicrobial disks $(6 \mathrm{~mm})$ were immersed in each test solution at varying concentrations for 15 seconds with excess solution allowed to drip off the disk. Subsequently, discs were placed on the inoculated 
plate at a rate of 1 disc per plate, followed by incubation at $30^{\circ} \mathrm{C}$ for 48 hours. Zones of inhibition were then measured and recorded in millimetres using a vernier calliper.

Inhibition of fungal growth: Inoculums of each test Candida spp. were prepared in sabouraud broth which was adjusted to meet the varying concentrations for each antifungal agent. The solutions were then seeded with a single colony of separate Candida test strains and incubated under rotary conditions $(125 \mathrm{rpm})$ at $30^{\circ} \mathrm{C}$ for 24 hours. Controls contained sabouraud dextrose broth only. Subsequently, $100 \mu \mathrm{l}$ of the diluted solution was aseptically serially diluted and spread on sabouraud dextrose agar plates in triplicate. All plates were incubated inverted for 48 hours at $30^{\circ} \mathrm{C}$. Surviving colonies were counted and reported as $\log 10 \mathrm{cfu} / \mathrm{ml}$ compared to an untreated control.

\section{Statistics}

All the experiments were performed three times with three plate replicates for each experimental data point providing a mean result for each test species and antifungal susceptibility (+/-standard deviation). The $\log 10$ inhibition of growth was calculated as the $\log 10$ of the ratio of the concentration $(\mathrm{cfu} / \mathrm{ml})$ of the non-treated $(\mathrm{N} 0)$ and treated $(\mathrm{N})$ samples $[\log 10(\mathrm{~N} 0 / \mathrm{N})]$. Student T tests were conducted to determine significance levels $(\mathrm{p}<0.05)$ of bacterial susceptibility to treatment using Minitab 16 (Minitab Ltd, Coventry, UK).

\section{Results}

Scanning electron microscopy was employed to confirm species identity and growth phase of Candida species. SEM (Figure 1) revealed typical colony morphology for both strains of yeast with well-defined structures and smooth membrane surfaces. Yeasts of the Candida genus are unicellular fungi which reproduce by generating daughter cells by a process of budding, separating at sites of septation. Typically, morphological characteristics of Candida budding cells manifest as oval, round, or cylindrical shapes as seen in Figure 1 (indicated by arrow). SEM images show the elongated cells of $C$. krusei compared to the round cells of $C$. albicans, both of which are attached to the adjacent cells, typical morphology for these species [10]. Figures $1 \mathrm{a}$ and $1 \mathrm{~b}$ depict actively reproducing cells of $C$. albicans and $C$. krusei skin isolates, respectively.

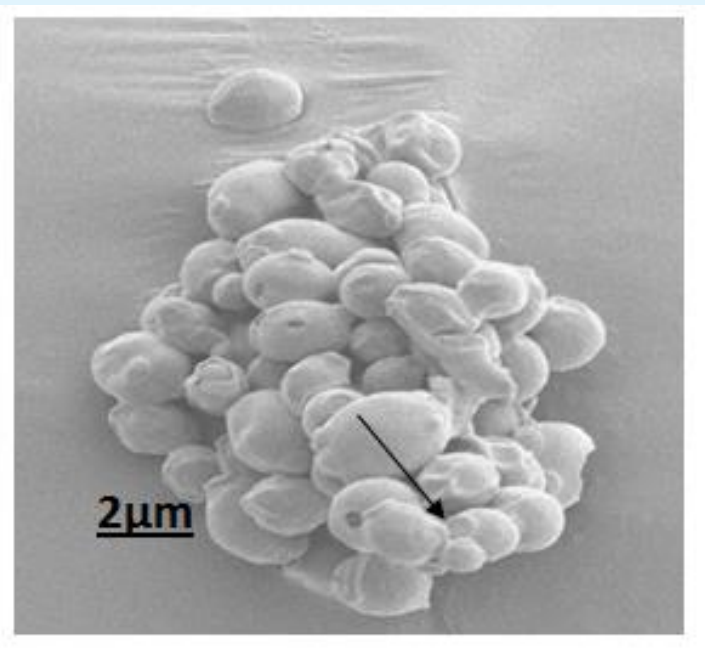

(a)

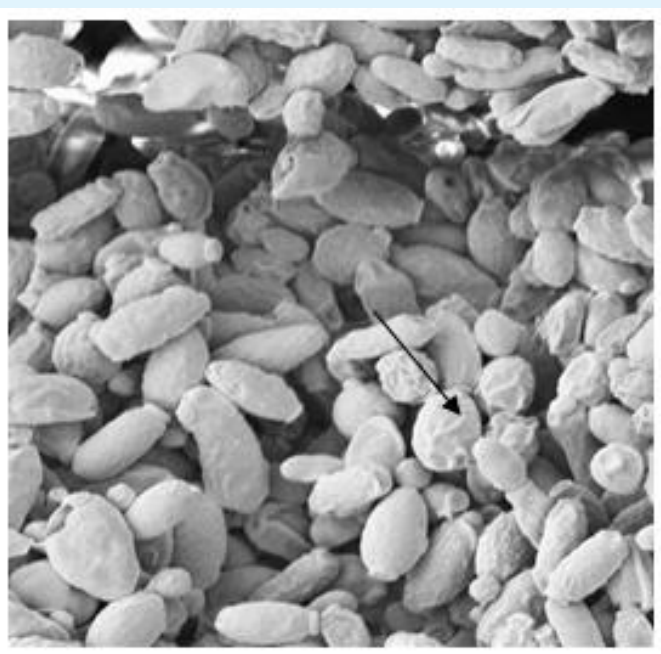

(b)

Figure 1: Scanning electron microscopy of a) C. albicans and b) C. krusei isolated from chronic cutaneous candidiasis of canine dermis, arrow indicating budding yeast cells.

Results show (Figure 2) the susceptibility of fungal test species to three common antifungal drugs as determined by the Kirby Bauer assay. Amp B (Figure 2a) provided a significant zone of inhibition at $2.5 \mathrm{ppm}$ which 


\section{Open Access Journal of Microbiology \& Biotechnology}

did not increase greatly with an increase in concentration for all test species. At $2.5 \mathrm{ppm}$ the kidney isolate of $C$. krusei proved most sensitive followed by C. albicans ATCC and $C$. krusei skin isolate. At concentrations of $12.5 \mathrm{ppm}$ all inhibition plateaued and did not increase at concentrations exceeding this, up to $50 \mathrm{ppm}$ (data not shown). All C. krusei strains showed similar susceptibility to treatment with the isolated species comparable to the reference strains (ATCC) at $6.25 \mathrm{ppm}$ however, a concentration of $12.5 \mathrm{ppm}$ provided a greater cell death of C. krusei ATCC and the kidney isolate. C. krusei skin isolate displayed some increase in resistance to Amp B as the concentration increased, showing that higher concentrations do not provide increased cell death. $C$. albicans skin isolate was the only species which showed an increase in zone diameter with an increase in Amp B concentration, increasing from 10 to $12 \mathrm{~mm}$ with a concentration increase to $12.5 \mathrm{ppm}$. At 6.25 and $12.5 \mathrm{ppm}$ both C. albicans strains proved more resistant to treatment than $C$. krusei. Fluconazole (Figure 2b) showed a slight decrease in cell death for C. albicans comparative to Amp B at $2.5 \mathrm{ppm}$ with similar results at 6.25 and 12.5 ppm. The skin isolate of $C$. albicans showed the same sensitivity to Amp B and fluconazole at all test concentrations, being more resistant than the ATCC strain to both antifungals. All C. krusei strains proved more sensitive to fluconazole than Amp B. C. krusei kidney isolate was more sensitive than the other krusei strains to fluconazole at $2.5 \mathrm{ppm}$ and this plateaued at $6.25 \mathrm{ppm}$ with no further dose dependent increase in cell death. Fluconazole gave a $12 \mathrm{~mm}$ zone of inhibition of $C$. krusei skin isolate at all test concentrations and proved the most sensitive test species to treatment. A dose dependent significant increase in zone diameter was observed for $C$. krusei kidney isolate, producing a $13.5 \mathrm{~mm}$ zone at 12.5 ppm. Both C. albicans strains and C. krusei kidney isolate showed greater sensitivity to caspofungin treatment than the two other drugs tested with a significant increase observed at 6.25 and $12.5 \mathrm{ppm}$ (Figure 2c). The zone of inhibition for the C. albicans ATCC strain increased from $11 \mathrm{~mm}$ at $2.5 \mathrm{ppm}$ to $14.5 \mathrm{~mm}$ at $6.25 \mathrm{ppm}$ and did not increase with a further increase in concentration. While $C$. albicans skin increased from $11 \mathrm{~mm}$ with Amp B to 15.5 $\mathrm{mm}$ following exposure to caspofungin at the same concentration (12.5 ppm). No significant difference in sensitivity to caspofungin was seen for $C$. albicans ATCC, C. albicans skin C. krusei ATCC at a concentration of 2.5 ppm but differences were observed above this concentration. C. krusei skin isolate proved more sensitive at this lower concentration with a significant increase in zone diameter at $12.5 \mathrm{ppm}$ compared to $6.5 \mathrm{ppm}$.

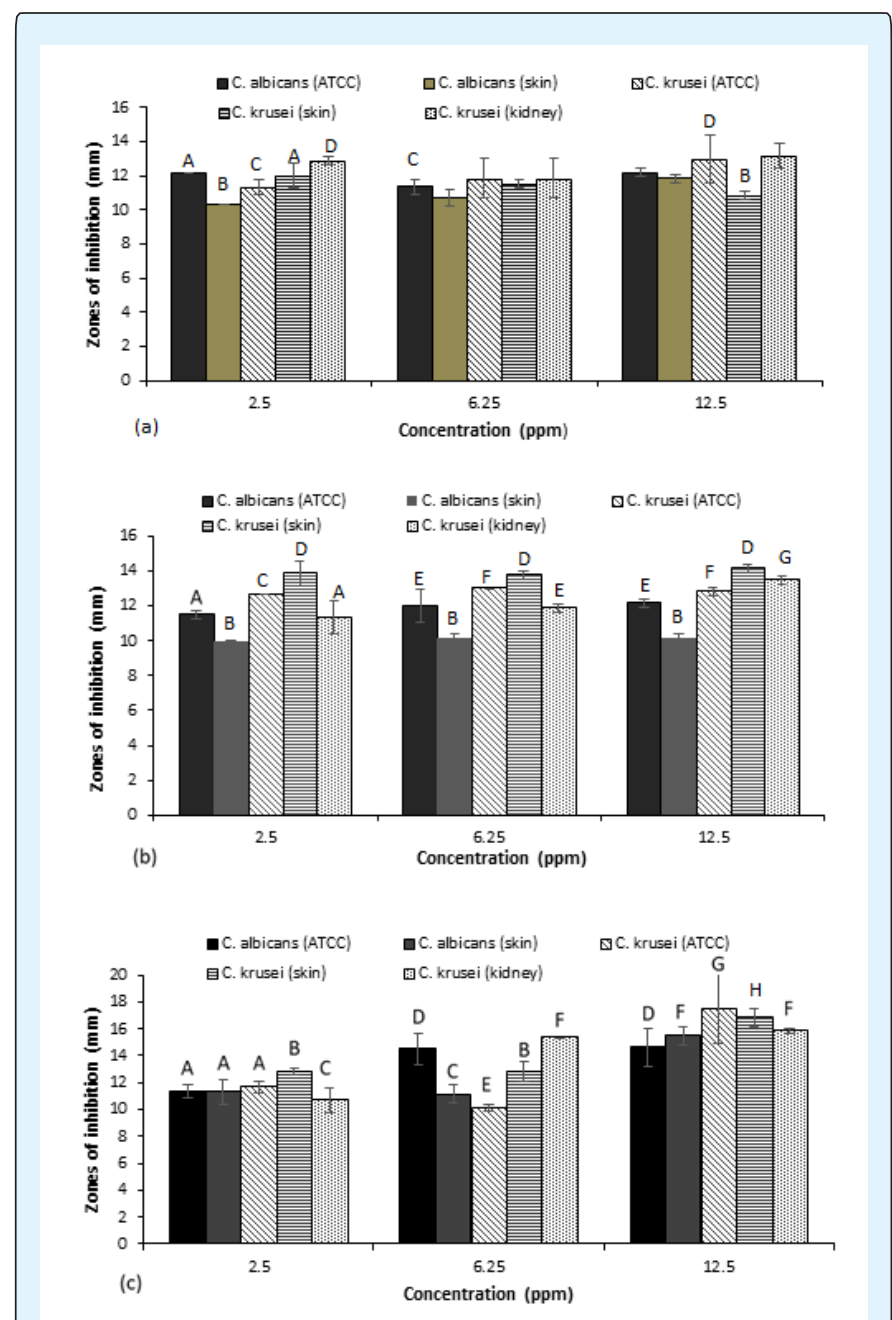

Figure 2: Antifungal susceptibility testing of Candia species (reference and isolate) to (a) amphotericin B, (b) fluconazole and (c) caspofungin as determined by the Kirby Bauer disk diffusion assay (+/- S.D). A, B, C, $D, E, F, G$ and $H$ denote significant difference at $p<0.05$.

The krusei kidney isolate proved most resistant to caspofungin at $2.5 \mathrm{ppm}$, with an increase in zone diameter as the antifungal concentration increased to a maximal $15.8 \mathrm{~mm}$ at $12.5 \mathrm{ppm}$. A concentration of $12.5 \mathrm{ppm}$ for all antifungal agents provided the greatest zones of inhibition for all test species. At this concentration of caspofungin, the order of sensitivity (from highest to lowest) is C. krusei ATCC, C. krusei skin, C. krusei kidney, C. albicans skin and C. albicans ATCC. Compared to Amp B where the order is as follows: C. krusei ATCC, C. krusei kidney, C. albicans (both strains) and C. krusei skin. Fluconazole provided the following order of sensitivity at 


\section{Open Access Journal of Microbiology \& Biotechnology}

this concentration: C. krusei skin, C. krusei kidney, C. krusei ATCC, C. albicans ATCC and C. albicans skin isolate. As displayed in Figure 2, caspofungin proved the most effective antifungal therapeutic agent of the three drugs tests, followed by fluconazole and Amp B at this optimal concentration of $12.5 \mathrm{ppm}$.

Figure 3 depicts the sensitivity of all test strains to the novel test compounds phendione, mitonafide and RBS. Results indicate that for phendione (Figure 3a), a $10 \mathrm{mM}$ $(2 \times 103 \mathrm{ppm})$ concentration was needed to produce zones of cell death comparable to the antifungal agents. Additionally, there was a dose dependent increase in cell death for all test species up to $50 \mathrm{mM}$, where the zones produced were significantly greater than those observed with the antifungal agents. At $10 \mathrm{mM}$, phendione provided a zone diameter of 15, 12, 14, 23.5 and $22 \mathrm{~mm}$ compared to $12.5 \mathrm{ppm}$ caspofungin giving a zone of $14.6,15.5,17.5$, 16.8 and $15.8 \mathrm{~mm}$ for $C$. albicans ATCC, C. albicans skin, $C$. krusei ATCC, C. krusei skin and C. krusei kidney isolate respectively. With an increase in concentration (to a max of $50 \mathrm{mM}$ ) however, the rate of cell death increased in a dose dependent manner (not observed with the antifungal therapeutics). Specifically, $50 \mathrm{mM}$ provided a zone diameter of 24, 19, 22.5, 31 and $32 \mathrm{~mm}$ for $C$. albicans ATCC, skin isolate, C. krusei ATCC, skin and kidney isolate respectively, a significant increase for all test species. C. albicans skin isolate proved most resistant to treatment with phendione, followed by C. krusei ATCC, C. albicans ATCC, C. krusei skin and kidney isolates.

All test strains showed a decreased sensitivity to mitonafide (Figure $3 \mathrm{~b}$ ) compared to phendione (and all the antifungal drugs) with a maximal zone diameter of 12 , 10, 2.5, 6 and $6 \mathrm{~mm}$ achieved for $C$. albicans ATCC, $C$. albicans skin, C. krusei ATCC, C. krusei skin and C. krusei kidney isolates respectively at $50 \mathrm{mM}$. At this concentration the order of sensitivity was C. albicans ATCC, skin isolate, C. krusei isolates with C. krusei ATCC proving most resistant to mitonafide. RBS (Figure 3c) provided consistent low level inactivation of all strains (comparative to phendione) as determined by the Kirby Bauer assay. Additionally, there was no significant increase in zone diameter with increasing RBS concentrations for all strains up to $0.4 \mathrm{mM}$, indicating that increasing the concentration of RBS does not provide increased antifungal activity when assessed by this method. A maximal zone of $12 \mathrm{~mm}$ was obtained at the highest concentration $(0.4 \mathrm{mM})$ for the most sensitive strain, C. krusei kidney isolate. The C. albicans strains proved slightly more resistant to RBS followed by $C$. krusei ATCC. As evident from figure 3, phendione provided the highest levels of antifungal activity exceeding that of all antifungal therapeutics tested (Figure 2) followed by RBS and lastly mitonafide (neither of which proved more yeasticidal than the antifungal drugs).
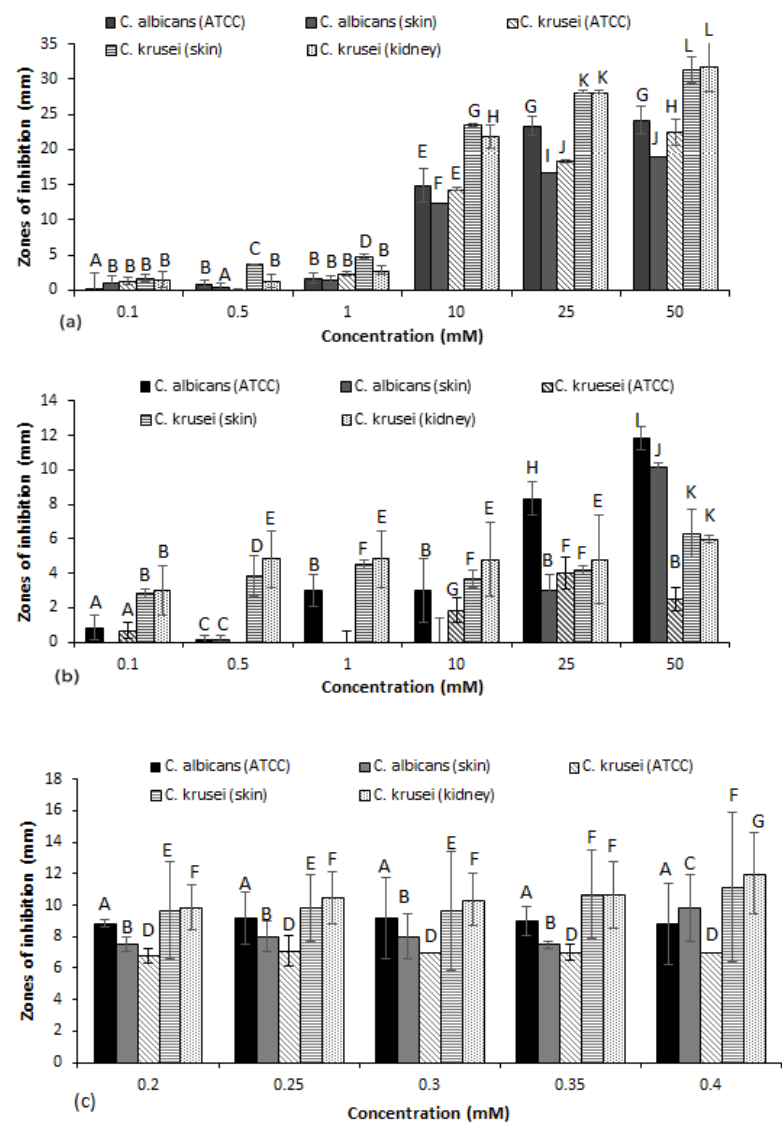

Figure 3: Antifungal susceptibility testing of Candia species (reference and isolate) to (a) phendione, (b) mitonafide and (c) RBS as determined by the Kirby Bauer disk diffusion assay (+/- S.D). A, B, C, D, E, F, G, $\mathrm{H}, \mathrm{I}, \mathrm{J}, \mathrm{K}$ and $\mathrm{L}$ denote significant difference at $\mathrm{p}<0.05$.

Figures 4 and 5 depict the log inhibition of cell growth of the antifungal therapeutics and novel compounds respectively. Amp B (Figure 4a) provided the greatest level of inhibition at a concentration of $25 \mathrm{ppm}$ and this did not increase in a dose dependent fashion. Similarly, as seen with the Kirby Bauer assay a plateau effect was evident at all concentrations exceeding $25 \mathrm{ppm}$. The ATCC strain of $C$. albicans proved most sensitive to inhibition with ca. $3 \log 10$ decrease in viable cell number compared 
to the untreated control followed by C. albicans skin $(2.5$ $\log 10)$, C. krusei ATCC (2.4 $\log 10)$, C. krusei kidney (1.9 $\log 10)$ and C. krusei skin (1.7 $\log 10)$.

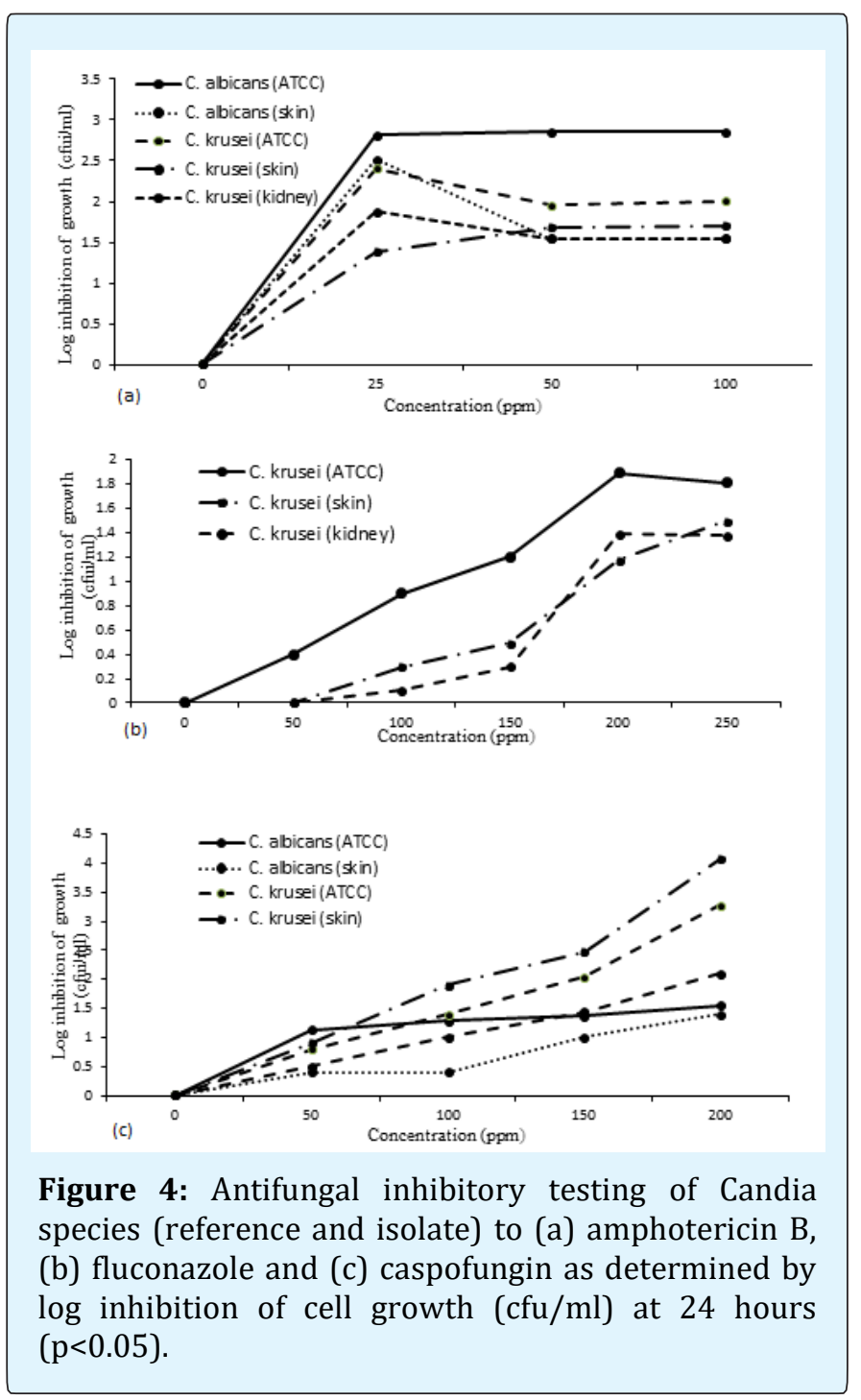

It must be noted however, that while $25 \mathrm{ppm}$ of AMP B provided the maximal inhibition found above for both $\mathrm{C}$. albicans strains, C. krusei ATCC and kidney isolate, 50 ppm was required however, for maximal inhibition of $C$. krusei skin isolate. C. krusei skin isolate proved the most resistant to AMP B inhibition with the lowest inhibition achieved overall. Fluconazole did not provide any inhibition of either C. albicans species at the concentration range tested indicating complete resistance to this drug. Furthermore, for all C. krusei strains (Figure 4b) a marginal decrease in cell inhibition is evident compared to AMP B, with a maximal 1.8, 1,5 and $1.4 \log 10$ $\mathrm{cfu} / \mathrm{ml}$ decrease in viable cell number achieved with 200 ppm fluconazole (significantly higher dose than AMP B). This data indicates a strong resistance to fluconazole for all test species. Caspofungin also proved more ineffective at inhibiting cell growth (Figure 4c) compared to AMP B, with $200 \mathrm{ppm}$ again required to achieve maximal inhibition. Caspofungin did inhibit both C. albicans strains, although to a lesser extent than AMP B, with a max inhibition of 1.5 and $1.4 \log 10 \mathrm{cfu} / \mathrm{ml}$ for the ATCC and skin strains respectively at $200 \mathrm{ppm}$. This concentration also provided a $3.2,4$ and $1.4 \log 10 \mathrm{cfu} / \mathrm{ml}$ inhibition of C. krusei ATCC, skin and kidney strains. Again all strains proved more resistant to caspofungin requiring a significantly higher concentration $(200 \mathrm{ppm})$ to obtain similar levels of inhibition to $25 \mathrm{ppm}$ AMP B. Caspofungin however, proved more effective than fluconazole for all Candida species. Therefore, for cell growth inhibition the order of effectiveness is AMP B, caspofungin and fluconazole, with the reference ATCC strains proving more sensitive than their isolated counterparts.

Phendione provided complete inhibition of both $C$. albicans strains and C. krusei skin isolate at $5 \mathrm{mM}$ with a ca. $7 \log 10 \mathrm{cfu} / \mathrm{ml}$ loss of viable cells compared to the untreated control. At concentrations exceeding this up to $50 \mathrm{mM}$ no increase in inhibition was possible, but some cell viability did manifest with a $1 \log 10$ recovery of cell numbers at $25 \mathrm{mM}$ (Figure 5a). C. krusei ATCC and kidney isolate proved more resistant to inhibition with a maximal $6.5 \log 10$ inhibition of both species with $10 \mathrm{mM}$ phendione. For both these strains however, there was significantly less viable cells evident at the higher concentrations. Mitonafide (Figure 5b) also provided significant inhibition of all test species compared to the untreated control. For all Candida species a maximal ca. $6.2 \log 10 \mathrm{cfu} / \mathrm{ml}$ inhibition of growth was achieved at 10 $\mathrm{mM}$ with $C$. krusei kidney isolate proving slightly more sensitive to mitonafide. (6.6 $\log 10$ inhibition). At higher concentrations inhibition did not increase and complete cell inhibition of any species was not achieved with this drug as seen with phendione. RBS (Figure 5c) also possesses the ability to inhibit fungal cell proliferation at the concentration range tested. For $C$. albicans strains, $C$. krusei ATCC complete inhibition (ca. $7 \log 10 \mathrm{cfu} / \mathrm{ml}$ ) at $0.4 \mathrm{mM}$ was achieved by RBS. C. krusei skin and kidney isolates proved more resistant to inhibition with a maximal 2.4 and $3.2 \log 10 \mathrm{cfu} / \mathrm{ml}$ inhibition occurring respectively at $0.4 \mathrm{mM}$ RBS. With increasing concentrations no further inhibition of yeast cells occurred. Based on inhibition of cell growth phendione 


\section{Open Access Journal of Microbiology \& Biotechnology}

proved the most antifungal agent of those tested providing significantly greater inhibition than AMP B, fluconazole and caspofungin.
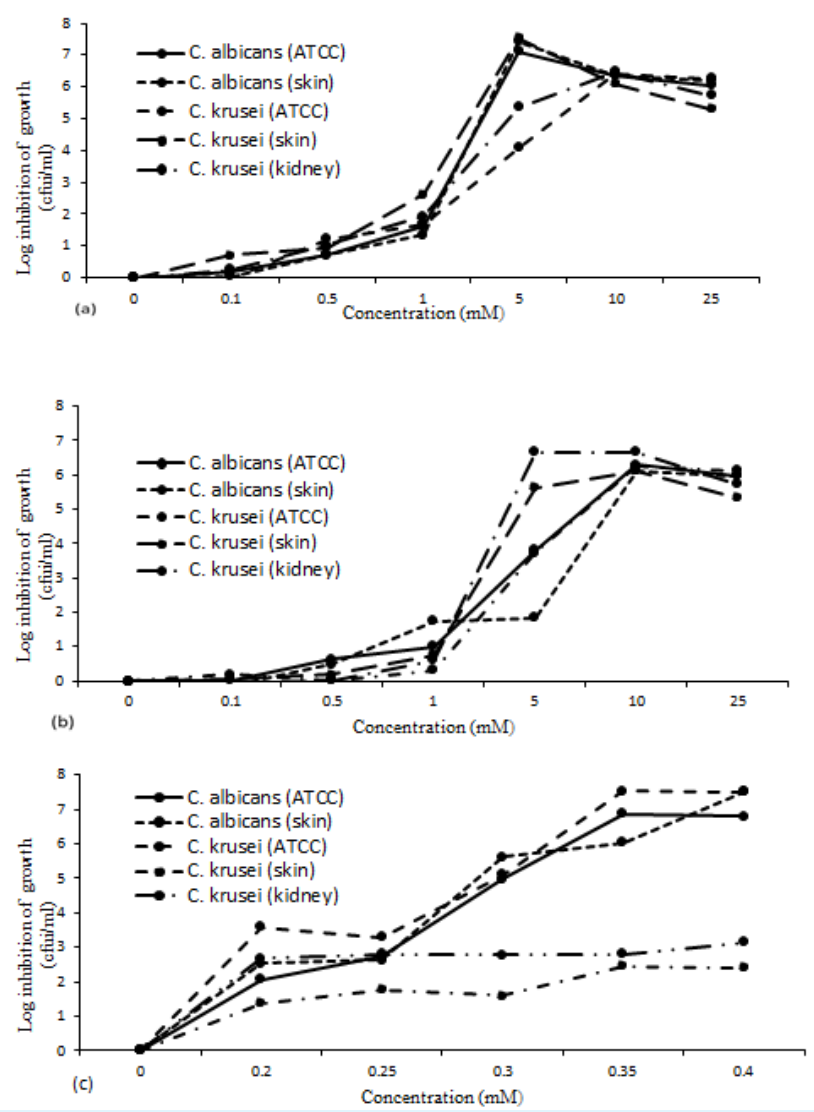

Figure 5: Antifungal inhibitory testing of Candia species (reference and isolate) to (a) phendione, (b) mitonafide and (c) RBS as determined by the log inhibition of cell growth $(\mathrm{cfu} / \mathrm{ml})$ at 24 hours $(\mathrm{p}<0.05)$.

\section{Discussion}

Chronic infections of companion animals represent an ongoing issue for veterinarians and a potential reservoir for zoonotic microbial species. Standard practice in treating cutaneous and systemic infections in canine animal's typical aims to target bacterial causative agents, where prescribing antibiotics such as cephalexin or amoxicillin is the primary course of action when patients present with a range of symptoms. Additionally, prednisolone is often prescribed to treat pyoderma or hypersensitivity in the animal. As demonstrated by this study for all three cases the causative agent was determined to be fungal with both Candida albicans and Candida krusei identified. This study suggests that the presence of a fungal causative agent is often initially overlooked in diagnosing infections in animals, allowing chronic conditions to manifest. Increasing evidence proposes that acquired resistance to antifungals such as amphotericin B and the azoles in Candida species may be an emerging and underdiagnosed risk. The findings of this study also suggest this, with caspofungin having the highest effect on all strains via the Kirby Bauer assay followed by fluconazole and lastly amphotericin B. For cell inhibition however, AMP B proved most effective followed by caspofungin and fluconazole. For treating human candidiasis AMP B is the primary drug of choice and in certain cases is combined with other antifungals such as azoles. Fluconazole is the azole of choice for local and systemic fungal infections. Candida krusei however, possess intrinsic resistance to this therapeutic [11]. Antifungal drug resistance can be measured as the minimum inhibitory concentration (MIC) that inhibits the growth of the fungus under standardized methods in vitro. MIC tests are used in resistance surveillance and in the comparative testing of new antimicrobial agents [12]. Complete fluconazole resistance was identified in this study for Candida albicans in the inhibitory assay and a decreased sensitivity was evident for all C. krusei strains under test. For this study no MIC was determined for any of the three antifungal agents up to $250 \mathrm{ppm}(0.25$ $\mathrm{mg} / \mathrm{ml}$ ), a significantly greater concentration than that reported by Eksi et al., 2013 [13]. This study reports less than $1 \mathrm{ppm}$ MICs for both Candida species exposed to AMP B and caspofungin and less than $65 \mathrm{ppm}$ for fluconazole. It must be noted however, that Eksi et al. (2013) [13] focused on human isolates using a micro dilution assay. Concentrations exceeding those under test are not realistic as antifungal drugs are known to cause toxicity to non-fungal cells. Consequently, success rates for AMP B use in veterinary medicine are difficult to assess due to the high proportion of patients incapable of tolerating an adequate antifungal dose [6]. AMP B toxicity manifests as vomiting, nausea, hypotension, nephrotoxicity and renal failure in chronic cases with daily doses of $35 \mathrm{mg}$ [14]. AMP B is not absorbed from the gastro intestinal tract when taken orally and is therefore given by IV for systemic fungal infections at a dose of 1 $\mathrm{mg} / 10 \mathrm{ml}$ or $0.1 \mathrm{mg} / \mathrm{ml}$ [15]. Caspofungin is administered by IV at a dose of $70 \mathrm{mg} /$ per day for the treatment of Candida infections with MICs ranging between 0.015 and 4 ppm [16] depending on the species. The increasing number of invasive and severe fungal infections is associated with the use of broad spectrum antibiotics and 


\section{Open Access Journal of Microbiology \& Biotechnology}

the increasing use of long term immunosuppressant drugs including chemotherapeutics [6]. The resistance seen in this study may relate to the administrating of antibiotic drugs to the animals before diagnosis was made. Candia species possess efflux pumps, a nonselective resistance mechanism which may have been activated in the presence of these xenobiotic compounds. Both reference ATCC strains proved more sensitive than their isolated counterparts in the inhibition assay suggesting that some environmental factor may have contributed to the resistance. The presence of this resistance in Candida species in companion animals is a serious concern as the possibility for human transmission is high risk. Virulence factors allow fungal species to colonise, invade and establish a pathogenic hold in the animal. The formation of fungal biofilms is once such virulence factor which resists the host immune system and antimicrobial therapy. The potential of cutaneous Candida infections to cross the dermis and enter the systemic circulation resulting in organ mycosis also increases it pathogenicity. The ability of Candida species to produce proteases, and hyphae allow for this tissue penetration and adherence to epithelia cells [17]. Furthermore, the prescribing of prednisolone to the patient may reduce the immune response of the animal and result in iatrogenic Cushing's or Addisons disease. A potential option for the treatment of such aggressive species is to combine or replace antifungal agents with novel products exhibiting antifungal activity such as those described herein. Phendione provided the highest level of antifungal activity for both assays and as such represents the best option from this study. Additionally, phendione provided complete inhibition of fungal growth for $C$. albicans with an MIC of $5 \mathrm{mM}$ identified. Studies have reported the antibacterial activity of phendione compounds against Pseudomonas planktonic and sessile cells [18]. The lipophilic nature of this drug is believed to contribute to its antimicrobial activity, where DNA binding may play a role in its toxicity. RBS is a polar compound which has long been known for its antibacterial activity. Studies report the antibacterial activity of RBS against Pseudomonas, Listeria and Clostridium due to lysis of the cell membranes [19]. RBS also shows potential for antifungal use, with an MIC of 0.4 mM identified for both $C$. albicans strains and C. krusei ATCC. Both $C$. krusei isolates showed a greater level of resistance however, with a max inhibition of 2.4 and 3.2 $\log 10 \mathrm{cfu} / \mathrm{ml}$ inhibition for C. krusei skin and kidney respectively. The findings of this study therefore demonstrate its aggressive antifungal activity against veterinary isolates of Candida species. The clinical result of Candida infection in humans and animals relates to many factors including the immune status of the host, drug pharmacokinetics, food-drug and drug-drug interactions. Consequently, drug studies in-cooperating all these factors need to be conducted including biocompatibility testing on relevant canine cell lines before a full profile is complete. Concentrations of phendione and RBS which provide antimicrobial activity at sub-cytotoxic levels in canine cells needs to be established using cell culture systems in vitro.

\section{Conclusion}

Fungal infections of companion animals result in animal morbidity, economic pressure and risk of zoonosis to animal owners. As human and animal relationships increase an amplified incidence of zoonotic disease is inevitable. Resistance to antifungal drugs is an ongoing problem particularly for immunosuppressed persons where chronic morbidity may lead to fatalities. The presence of resistant species in companion animals such as those described herein, represents an often unrecognised route of disease transmission. Candida species represent a significant portion of infective agents of disease particularly in dermal cases, with a potential for penetration and systemic infection. The development of new treatment options is therefore essential for both animal and human incidents of infection in order to ensure public health safety and quality of life. Two drugs used in this study have shown potential as antifungal agents and should be further analysed to determine their ability to remain cell toxic irrespective of fungal virulence factors and resistance mechanisms.

\section{Conflict of Interest}

The authors declare there is no conflict of interest.

\section{References}

1. Messenger AM, Barnes AN, Gray GC (2014) Reverse Zoonotic Disease Transmission (Zooanthroponosis): A Systematic Review of Seldom-Documented Human Biological Threats to Animals. PLoS ONE 9(2).

2. Pal M (2017) Morbidity and Mortality Due to Fungal Infections. Journal of Applied Microbiology and Biochemistry 1(2).

3. Kanafani ZA, Perfect JR (2008) Resistance to Antifungal Agents: Mechanisms and Clinical Impact. Clinical Infectious Diseases 46: 120-128. 


\section{Open Access Journal of Microbiology \& Biotechnology}

4. Blanco JL, Garcia ME (2008) Immune response to fungal infections. Veterinary Immunology and Immunopathology 125: 47-70.

5. Alvarez-Perez S, Garcia ME, Cutuli MT, Fermin ML, Daza MA, et al. (2016) Acquired multi-azole resistance in Candida tropicalis during persistent urinary tract infection in a dog. Med Mycol Case Rep 11: 9-12.

6. Wiebe V, Karriker M (2005) Therapy of Systemic Fungal Infections: A Pharmacologic Perspective. Clini Tech Small Anim Pract 20(4): 250-257.

7. Yu H, Liu X, Wang C, Qiao X, Wu S, et al. (2016) Assessing the potential of four cathelicidins for the management of mouse candidiasis and Candida albicans biofilms. Biochimie 121: 268-277.

8. Bumroongthai $\mathrm{K}$, Chetanachan $\mathrm{P}$, Niyomtham $\mathrm{W}$, Yurayart C, Prapasarakul N (2016) Biofilm production and antifungal susceptibility of cocultured Malassezia pachydermatis and Candida parapsilosis isolated from canine seborrheic dermatitis. Medical Mycology 54(5): 544-549.

9. Braña MF, Castellano JM, Roldan CM, Santos A, Vazquez D, et al. (1980) Synthesis and mode (s) of action of a new series of imide derivatives of 3-nitro1, 8 naphthalic acid. Cancer chemotherapy and pharmacology 4(1): 61-66.

10. Kim KS, Kim YS, Han I, Kim MH, Jung MH, et al. (2011) Quantitative and Qualitative Analyses of the Cell Death Process in Candida albicans Treated by Antifungal Agents. PLoS ONE 6(12): 28176.

11. Pfaller MA, Diekema DJ, Gibbs DL, Newell VA, Nagy E, et al. (2008) Candida krusei, a Multidrug-Resistant Opportunistic Fungal Pathogen: Geographic and Temporal Trends from the ARTEMIS DISK Antifungal Surveillance Program, 2001 to 2005. J Clin Microbiol 46(2): 515-521.
12. EUCAST (2003) Determination of minimum inhibitory concentrations (MICs) of antibacterial agents by broth dilution. European Committee for Antimicrobial Susceptibility Testing (EUCAST) of the European Society of Clinical Microbiology and Infectious Diseases (ESCMID).

13. Eksi F, Gayyurhan ED, Balci I (2013) In Vitro Susceptibility of Candida Species to Four Antifungal Agents Assessed by the Reference Broth Microdilution Method. The Scientific World Journal.

14. Laniado-Laborín R, Cabrales-Vargas MN (2009) Amphotericin B: side effects and toxicity. Revista Iberoamericana de Micología 26(4): 223-227.

15. WHO, 2018. http://apps.who.int/medicinedocs/en/d/Js2215e/9. 3.html

16. Letscher-Bru V, Herbrecht R (2003) Caspofungin: the first representative of a new antifungal class. Journal of Antimicrobial Chemotherapy 51(3): 513-521.

17. Raz-Pasteur A, Ullmann Y, Berdicevsky I (2011) The Pathogenesis of Candida Infections in a Human Skin Model: Scanning Electron Microscope Observations. ISRN Dermatology.

18. Viganor L, Galdino ACM, Nunes APF, Santos KRN, Branquinha $\mathrm{MH}$, et al. (2006) Anti-Pseudomonas aeruginosa activity of 1,10-phenanthroline-based drugs against both planktonic- and biofilm-growing cells. J Antimicrob Chemoth 71(1): 128-134.

19. Hamilton-Brehm SC, Schut GJ, Adams MWW (2009) Antimicrobial Activity of the Iron-Sulfur Nitroso Compound Roussin's Black Salt $\left[\mathrm{Fe}_{4} \mathrm{~S}_{3}(\mathrm{NO})_{7}\right]$ on the Hyperthermophilic Archaeon Pyrococcus furiosus. Appl Environ Microbiol 75(7): 1820-1825. 